\title{
Density Fluctuation Measurement for Studying the Effect of Potential and Electric Field Formation in GAMMA 10
}

\author{
Masayuki YOSHIKAWA, Toshiaki MATSUMOTO, Yoriko SHIMA, Yoshiaki MIYATA, Hideto \\ KAKIUCHI, Masanori MIZUGUCHI, Makoto ICHIMURA, Tsuyoshi IMAI, Akiyosi ITAKURA, \\ Isao KATANUMA, Junko KOHAGURA, Yoshinori TATEMATSU, Yousuke NAKASHIMA, \\ Tomoharu NUMAKURA, Mafumi HIRATA, Hitoshi HOJO, Yuusuke KUBOTA, Ryutaro MINAMI, \\ Takayuki KOBAYASHI, Yuusuke YAMAGUCHI, Hiroshi SAIMARU, Yuta HIGASHIZONO, \\ Teruji CHO and GAMMA 10 Group \\ Plasma Research Center, University of Tsukuba, Tsukuba, Ibaraki 305-8577, Japan
}

(Received 4 December 2006 / Accepted 5 October 2007)

\begin{abstract}
Advances in potential formation have led to remarkable discoveries on the effects of radial electric field distribution on turbulence suppression and transverse loss reduction. In order to study the improvement in plasma confinement because of the formation of plasma confinement potential, we constructed a multi-channel microwave interferometer system that can measure the density and density fluctuation radial profiles in a single plasma shot. We obtained clear density fluctuation suppression by the formation of the plasma confinement potential. Therefore, we have a powerful diagnostic tool with which to study the improvement in plasma confinement.
\end{abstract}

(C) 2007 The Japan Society of Plasma Science and Nuclear Fusion Research

Keywords: tandem mirror GAMMA 10, potential and electric field, $E_{\mathrm{r}}$ shear, multi-channel microwave interferometer

DOI: $10.1585 /$ pfr. 2.048

\section{Introduction}

The tandem mirror GAMMA 10 (Fig. 1) utilizes an electron cyclotron resonance heating (ECRH) for forming a confinement potential. Advances in the potential formation have led to remarkable discoveries on the effects of radial electric fields gradient $\mathrm{d} E_{\mathrm{r}} / \mathrm{d} r$ on turbulence suppression and transverse loss reduction. We obtained a preferable dependence of a weak decrease in ion-confining potential in the central cell $\phi_{\mathrm{c}}$ with increasing electron density in the central cell $n_{\mathrm{c}}$ ranging to $\sim 10^{13} \mathrm{~cm}^{-3}$ along with the recovery of $\phi_{\mathrm{c}}$ with increasing plug ECRH powers $\left(\mathrm{P}_{\mathrm{ECH}}\right)$ is obtained [1-4]. This leads to suppress intermittent turbulent vortex-like structures near the layer in the central cell, and results in electron temperature $T_{\mathrm{e}}$ and ion temperature $T_{\mathrm{i}}$ rises surrounded by the layer having a localized bumped ambipolar potential $\Phi_{\mathrm{C}}$. The radial transport barrier is accounted for by the formation of a strong $E_{\mathrm{r}}$ gradient or peaked vorticity $W$ with a reversal in direction of $E_{\mathrm{r}} \times B$ sheared flow near the $\Phi_{\mathrm{C}}$ peak for the turbulence suppression [2-8]. To study this improvement in plasma confinement because of the formation of confinement potential, we have constructed a new multi-channel microwave interferometer [9]. This system can measure electron density and density fluctuation radial profiles in a single plasma shot. It is therefore a powerful tools with which to understand $E_{\mathrm{r}}$ shear effects.

In this paper, we show the multi-channel microwave

author's e-mail: yosikawa@prc.tsukuba.ac.jp

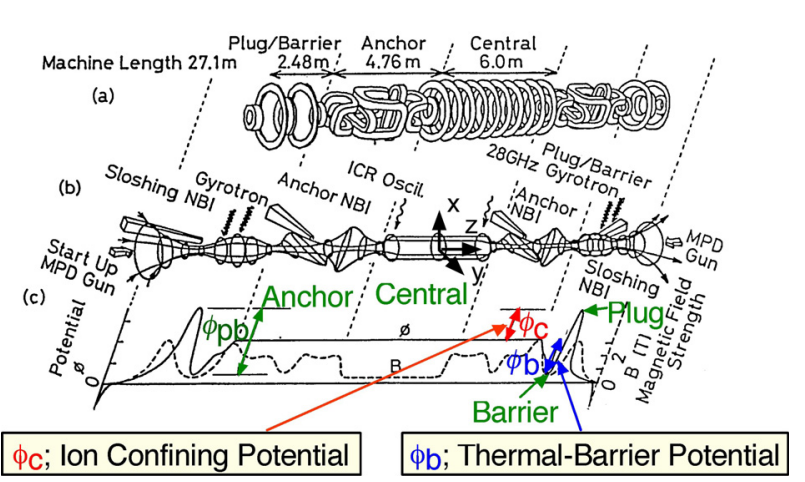

Fig. 1 Schematic of GAMMA 10 tandem mirror.

interferometer and clear results for density fluctuation suppression by the effect of the electric field formation and potential formation with applying the plug ECRH.

\section{Experimental Apparatus}

GAMMA 10 is an effectively axisymmetrized minimum- $B$ anchored tandem mirror with a thermal barrier at both end-mirrors [1-4,6,9-16]. The device consists of an axisymmetric central mirror cell $\left(L_{\mathrm{z}}=6 \mathrm{~m}\right.$, limiter diameter $=36 \mathrm{~cm}$, and magnetic fields $B_{\mathrm{z}}=B_{\mathrm{m}}$ at the midplane of $0.405 \mathrm{~T}$, with a mirror ratio $R_{\mathrm{m}}$ of 5.2), anchor cells with minimum- $B$ configuration, and plug/barrier cells ( $L_{\mathrm{z}}=2.5 \mathrm{~m}, B_{\mathrm{m}}=0.497 \mathrm{~T}$, and $R_{\mathrm{m}}=6.2$ for standard operations) with axisymmetric mirrors. In the tandem mirror 
GAMMA 10, the plasma confinement is achieved not only by a magnetic mirror configuration but also by high potentials at both end regions. The primary plasma confined in GAMMA 10 is produced and then heated by ion cyclotron range of frequency (ICRF: $30 \mathrm{~kW}$ at $6.36 \mathrm{MHz}$ for centralcell hot-ion production, and $30 \mathrm{~kW}$ at 9.9 or $10.3 \mathrm{MHz}$ for anchor-cell stabilization) power deposition. The potentials are produced by means of ECRH at the plug/barrier region $(150 \mathrm{~kW}$ at $28 \mathrm{GHz}$ at the barrier region, and from 250 to $380 \mathrm{~kW}$ at the plug ECRH area). Neutral beam injection (NBI: $25 \mathrm{kV} / 15 \mathrm{~A}$ ) is also utilized at the plug/barrier cell to produce the sloshing ion. In addition, to produce higher plasma density operation, we performed pellet injection experiments. Before injecting the hydrogen submillimeter pellet, the typical electron density, and electron and ion temperatures were about $2 \times 10^{12} \mathrm{~cm}^{-3}, 0.08 \mathrm{keV}$ and $5 \mathrm{keV}$, respectively. The axes $x, y$, and $z$ are shown in Fig. 1. The mid plane of the GAMMA 10 device is set to $z=0$. The central axis of GAMMA 10 is $x$ and $y=0$.

Various fluctuation diagnostics are used, one of which is a new multi-channel microwave interferometer (Fig. 2) for measuring the plasma density and density fluctuation profiles in a single plasma shot [9]. The interferometer was designed based on the principles of Gaussian-beam propagation theory and ray-tracing code. The system was configured as a heterodyne interferometer consisting of a $70 \mathrm{GHz}(1 \mathrm{~W})$ IMPATT oscillator and a $150 \mathrm{MHz}$ oscillator. The probe microwave beam is injected into the plasma without a lens system from the upper port of GAMMA 10. The probe beam spreads and is received by the six horns mounted at the measuring position of $y=-1.7 \mathrm{~cm}$ (channel 1), $-3.7 \mathrm{~cm}$ (channel 2), $-6.3 \mathrm{~cm}$ (channel 3), $-9.3 \mathrm{~cm}$ (channel 4), $-11.4 \mathrm{~cm}$ (channel 5), and $-14.4 \mathrm{~cm}$ (channel 6) at the bottom, outside the GAMMA 10's port. The

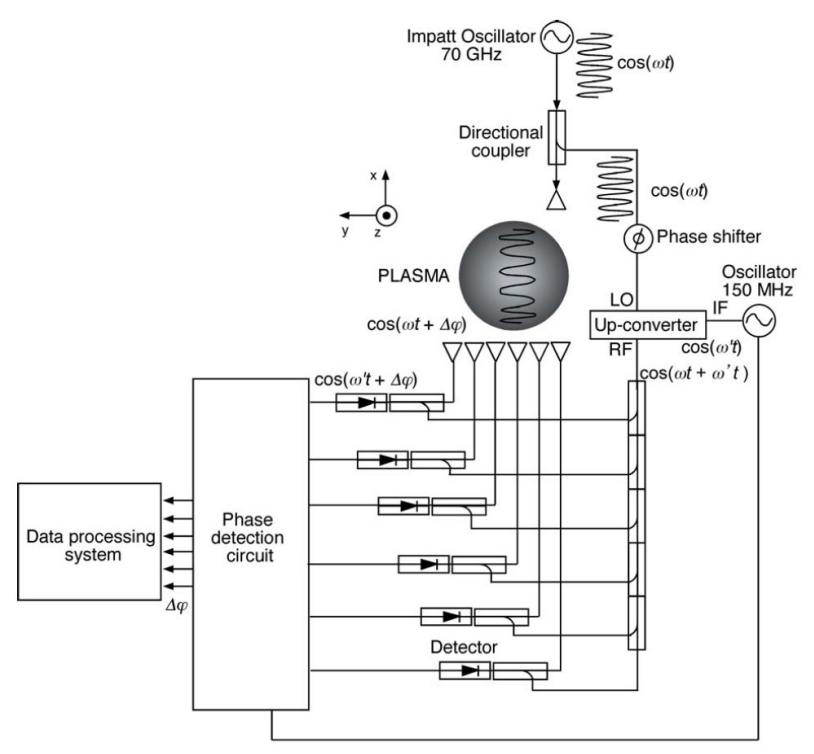

Fig. 2 Schematic diagram of the multi-channel microwave interferometer. spatial resolution of the system is approximately $3 \mathrm{~cm}$, and the line-integrated electron density of each position is calculated. The Abel inversion technique is used for obtaining the electron density radial profile. We combined the data of the movable interferometer at the measured position of $y=6 \mathrm{~cm}$ with those of the multi-channel interferometer. Other diagnostic techniques are an ultrashort-pulse reflectometry system for the local density fluctuation measurements [13], two sets of 50-channel soft X-ray tomography detectors $[2,3]$ using microchannel plates (MCP) in the central-cell midplane, several original semiconductors in various cells for $T_{\mathrm{e}}$ and $T_{\mathrm{i}}$ diagnostics, and eight Langmuir probes [2] for wave-phasing and coherence diagnosis. A gold neutral $\left(\mathrm{Au}^{0}\right)$ beam probe (GNBP) [14] and eight sets of ion-energy-spectrometer (IES) arrays [15] are used for measuring the potential. Energetic electron currents are observed with a radially movable conventional end-loss analyzer (ELA) and ELA arrays [16] at the west end.

\section{Density Fluctuation Measurements}

The plasma is produced at $50.5 \mathrm{~ms}$ and sustained by ICRF. Barrier ECRH ( $150 \mathrm{~kW})$ is applied between 155 and $175 \mathrm{~ms}$ to create thermal barrier potentials, and plug ECRH ( $260 \mathrm{~kW}$ at the east plug and $270 \mathrm{~kW}$ at the west plug) is applied between 160 and $170 \mathrm{~ms}$ to create confining potentials. NBI $(20 \mathrm{~kW} / 15 \mathrm{~A})$ is injected between 159 and $163 \mathrm{~ms}$. The electron density in the central cell is measured in a single plasma shot by the movable interferometer and the newly installed multi-channel microwave interferometer. The pellet is injected at $163.5 \mathrm{~ms}$. We concentrated on the effect on potential confinement, due to the application of plug ECRH during formation of the plug potential. Figure 3 shows the line density and diamagnetism at between 152 and $163 \mathrm{~ms}$. The diamagnetism increases on application of plug ECRH, and the electron density slightly increases on applying barrier ECRH. Fluctuation of the

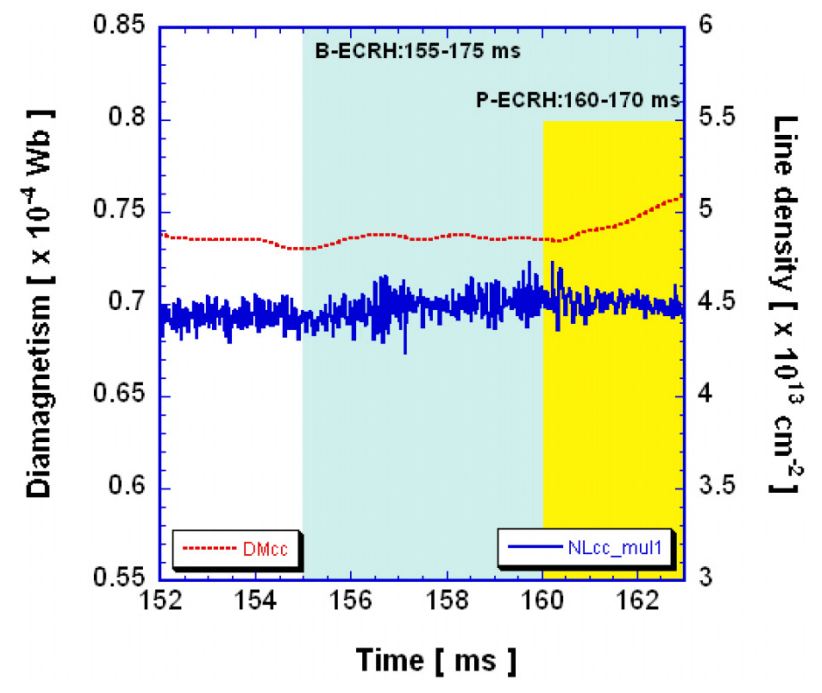

Fig. 3 Line density and diamagnetism between 152 and $163 \mathrm{~ms}$. 


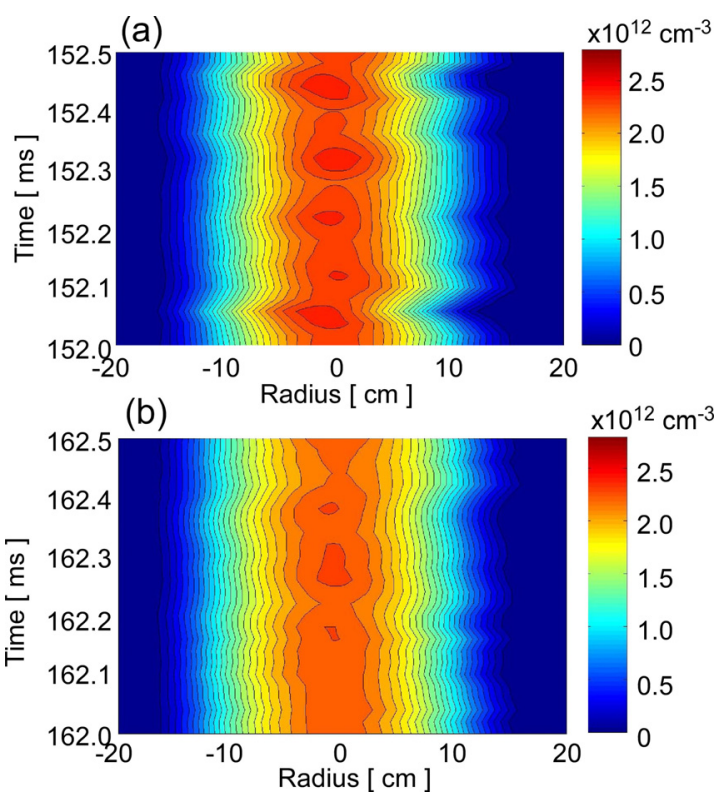

Fig. 4 Electron density radial profiles (a) before applying plug ECRH at $152-152.5 \mathrm{~ms}$, and (b) during plug ECRH at $162-162.5 \mathrm{~ms}$.

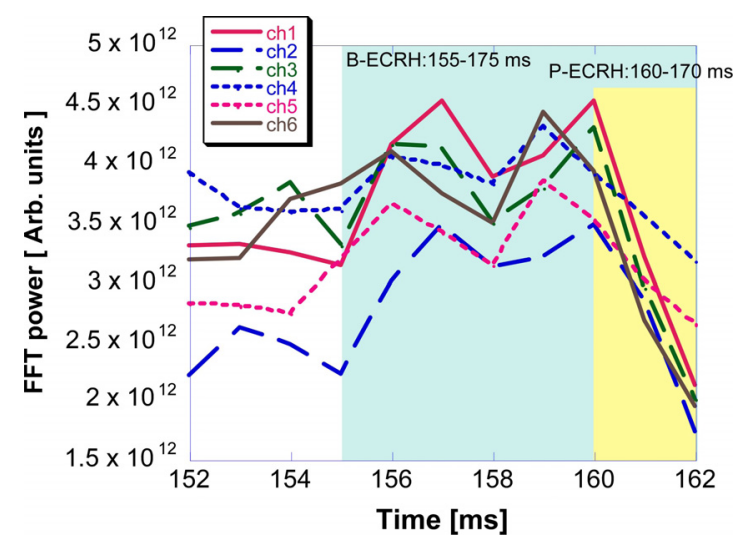

Fig. 5 Time-dependent FFT fluctuation powers of the lineintegrated densities, measured at each position using the multi-channel interferometer.

line density decreases on application of plug ECRH. Figure 4 (a) and (b) show the electron density radial profiles before the application of plug ECRH at 152-152.5 ms and the profiles during the application of plug ECRH at 162$162.5 \mathrm{~ms}$, respectively. The electron density is approximately $2.3 \times 10^{12} \mathrm{~cm}^{-3}$. Radial density fluctuation is small, as shown in Fig. 4(b), while clear radial density fluctuation is shown in Fig. 4 (a). It is therefore shown that a clear density fluctuation suppression is obtained because of the formation of the confinement potential on application of plug ECRH.

Figure 5 shows the time-dependent Fast Fourier Transformed (FFT) fluctuation powers of the lineintegrated densities, measured at every position using the multi-channel interferometer. The FFT amplitudes of channels 4 and 5 exhibit a slight fluctuation suppression
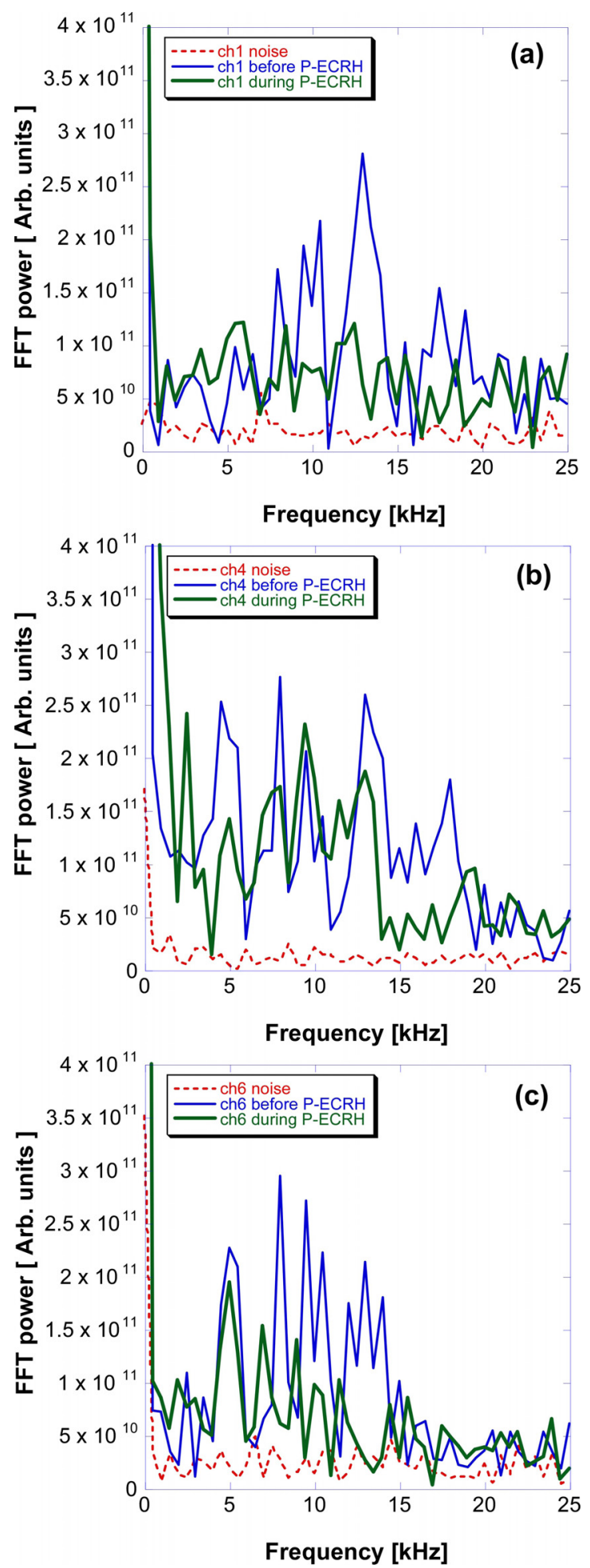

Fig. 6 FFT spectra at noise-level (dotted lines), before (hair lines) and during (bold lines) application of plug ECRH for (a) channel 1, (b) channel 4, and (c) channel 6.

during the plug ECRH period when compared to other channels. Figure 6(a), (b), and (c) show the FFT spectra at noise-level (dotted lines), before (hair lines: at 152$153 \mathrm{~ms}$ ), and during (bold lines: at 162-163 ms) the application of plug ECRH, for channels 1, 4, and 6, respectively. A number of strong peaks are observed in the spectrum of each channel before application of plug ECRH. 


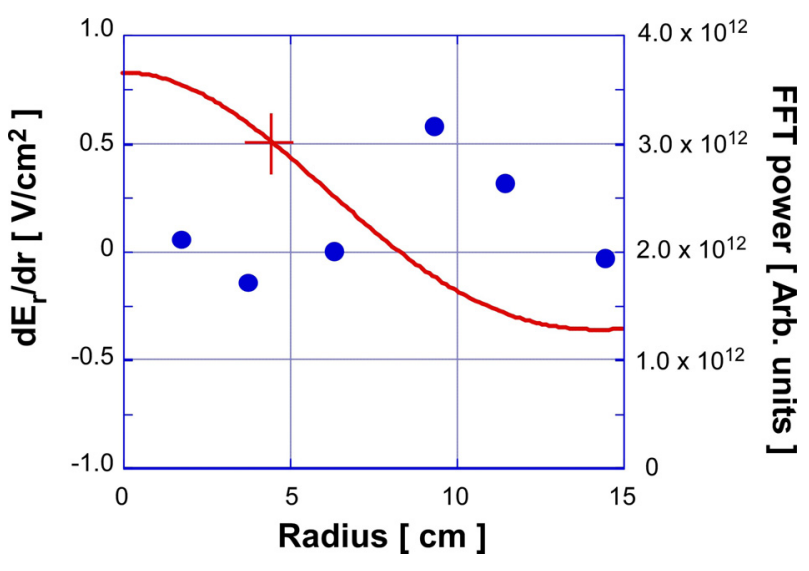

Fig. 7 The solid line shows the $\mathrm{d} E_{\mathrm{r}} / \mathrm{d} r$ radial profile during application of plug ECRH at $162.3-163.3 \mathrm{~ms}$, as measured using the GNBP. The circles show the radial line density fluctuations of FFT power at $162-163 \mathrm{~ms}$.

The coherent mode, near the frequency of $13 \mathrm{kHz}$, corresponds to diamagnetic drift. During the application of plug ECRH, the spectrum exhibit lower fluctuation in all observed channels except for channels 4 and 5 . The measured position of channels 4 and 5 corresponds to the lower value of the $\mathrm{d} E_{\mathrm{r}} / \mathrm{d} r$ position. In Fig. 7, we show the $\mathrm{d} E_{\mathrm{r}} / \mathrm{d} r$ radial profiles during application of plug ECRH at 162.3$163.3 \mathrm{~ms}$, measured using the GNBP (solid line). The figure also shows radial line density fluctuations of FFT power at $162-163 \mathrm{~ms}$, represented by circles. In the radial positions where the $\mathrm{d} E_{\mathrm{r}} / \mathrm{d} r$ is approximately zero, the linedensity fluctuation powers are larger than those in the other positions.

We also carried out a preliminary measurement of the local density fluctuation using an ultrashort-pulse reflectometry system. Figure 8 shows one of the FFT spectra, determined using this system, at noise-level (dotted lines), before (hair lines: 152-153 ms), and during (bold lines: $162-163 \mathrm{~ms}$ ) the application of plug ECRH for the timeof-flight signals at $7 \mathrm{GHz}$. The cut-off layer is located at about $r=12 \mathrm{~cm}$. The results indicate local density fluctuation suppression by the formation of confinement potential on application of plug ECRH.

These are the first clear results on density fluctuation suppression because of confinement potential formation by $\mathrm{d} E_{\mathrm{r}} / \mathrm{d} r$ shear formation. However, this is middle $\mathrm{d} E_{\mathrm{r}} / \mathrm{d} r$ shear-produced plasma. Therefore, we plan to study density fluctuation suppression mechanisms in higher $\mathrm{d} E_{\mathrm{r}} / \mathrm{d} r$ shear-produced plasma in further detail.

\section{Summary}

We have constructed a new multi-channel microwave interferometer, in order to study the effect of confinement potential and electric field shear formation. Fluctuation suppression by the formation of confinement potential has

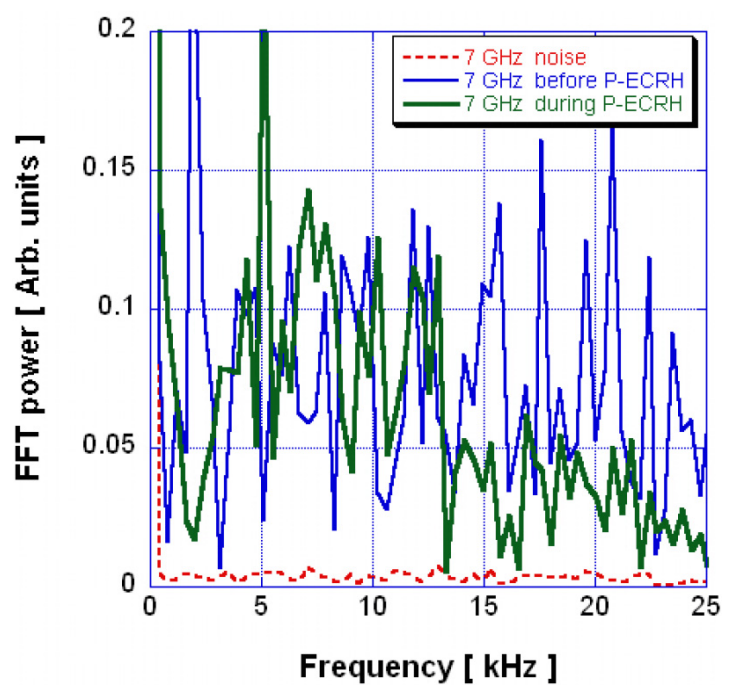

Fig. 8 One of the FFT spectra at noise-level (dotted lines), before (hair lines: at $152-153 \mathrm{~ms}$ ), and during (bold lines: $162-163 \mathrm{~ms}$ ), the application of plug ECRH, for timeof-flight signals of the ultrashort-pulse reflectometry at $7 \mathrm{GHz}$.

been clearly observed using, for the first time, a multichannel microwave interferometer. Therefore, we have a powerful tool with which to study the improvements in plasma potential confinement.

\section{Acknowledgments}

The authors would like to thank members of the GAMMA 10 group of the University of Tsukuba for their collaboration. This work was partly supported by the Ministry of Education, Culture, Sports, Science and Technology, and a Grant-in-Aid for Scientific Research in Priority Areas, No 16082203. This work was conducted with the support and under the auspices of the NIFS Collaborative Research Program, No NIFS04KOAP009.

[1] T. Cho et al., Phys. Rev. Lett. 86, 4310 (2001).

[2] T. Cho et al., Phys. Rev. Lett. 94, 085002 (2005).

[3] T. Cho et al., Nucl. Fusion 45, 1650 (2005).

[4] T. Cho et al., 21th IAEA Fusion Energy Conf. (Chengdu, 2006) IAEA/EX/P7-14.

[5] R.F. Post, Nucl. Fusion 27, 1579 (1987).

[6] A. Mase et al., Phys. Rev. Lett. 64, 2281 (1990).

[7] M.B. Chaudhry et al., J. Phys. Soc. Jpn. 57, 3043 (1988).

[8] M. Kwon et al., Trans. Fusion Sci. Tech. 43, 23 (2003).

[9] M. Yoshikawa et al., Rev. Sci. Instrum. 77, 10 E906 (2006).

[10] M. Yoshikawa et al., Trans. Fusion Technol. 35, 273 (1999).

[11] M. Yoshikawa et al., Surface Rev. Lett. 9, 555 (2002).

[12] M. Yoshikawa et al., Trans. Fusion Sci. Tech. 43, 189 (2003).

[13] M. Yoshikawa et al., J. Korean Phys. Soc. 49, S113 (2006).

[14] K. Ishii et al., Rev. Sci. Instrum. 60, 3270 (1989).

[15] M. Yoshida et al., Rev. Sci. Instrum. 74, 1909 (2003).

[16] T. Cho et al., Phys. Rev. A 45, 2532 (1992). 